Philosophie ANTIQUE
Philosophie antique

Problèmes, Renaissances, Usages

14 | 2014

Le devoir

\title{
Magali ANNÉE, Parménide : Fragments Poème, précédé de Énoncer le verbe être
}

\section{Annick Stevens}

\section{OpenEdition}

\section{Journals}

Édition électronique

URL : https://journals.openedition.org/philosant/828

DOI : 10.4000/philosant.828

ISSN : 2648-2789

\section{Éditeur}

Éditions Vrin

\section{Édition imprimée}

Date de publication : 1 novembre 2014

Pagination : $322-325$

ISBN : 978-2-7574-0855-1

ISSN : $1634-4561$

\section{Référence électronique}

Annick Stevens, « Magali annÉE, Parménide : Fragments Poème, précédé de Énoncer le verbe être », Philosophie antique [En ligne], 14 | 2014, mis en ligne le 01 novembre 2018, consulté le 03 décembre 2022. URL : http://journals.openedition.org/philosant/828 ; DOI : https://doi.org/10.4000/philosant. 828

\section{(c) (i) (9)}

Creative Commons - Attribution - Pas d'Utilisation Commerciale - Pas de Modification 4.0 International - CC BY-NC-ND 4.0

https://creativecommons.org/licenses/by-nc-nd/4.0/ 
précédents, une grande attention est accordée à la réception : une vingtaine de pages fort intéressantes sont consacrées à Platon et la tradition arabe, et l'on trouve même une rubrique sur Plutarque et la culture européenne.

À une époque où les projets à court terme sont privilégiés, un tel travail collectif d'érudition mené sur une trentaine d'années n'obtiendrait pas actuellement de financement et il ne pourrait pas voir le jour. C'est pourtant sans doute l'une des publications les plus largement utiles, pour l'étudiant comme pour le chercheur. On n'en est que plus admiratif devant la somme de travail réalisée par le maître d'œuvre et ses contributeurs, et l'on se sent redevable à leur égard d'avoir mis à notre disposition un outil de travail infiniment précieux.

Pierre PONTIER

Université Paris-Sorbonne Paris 4

Magali ANNÉE, Parménide: Fragments Poème, précédé de Énoncer le verbe être, Paris, Librairie philosophique J. Vrin, 2012 (Bibliothèque des textes philosophiques), 215 pages, ISBN 978-2-6116-2414-0.

Une étude qui se situe exclusivement sur le terrain linguistique est bien placée pour attirer l'attention de l'interprète de Parménide, avide d'un éclairage nouveau sur un texte tellement commenté qu'il semble ne plus être possible d'y apporter quoi que ce soit. Le point de vue du grammairien pourrait en effet révéler certaines subtilités jusque-là ignorées, en particulier s'il ne cherche pas à mettre la grammaire au service d'une signification philosophique (comme on l'a déjà fait abondamment) mais à limiter la signification à la grammaire même. Autrement dit : que peut-on tirer de la lettre d'un texte avant d'y introduire toute notion héritée de l'histoire de la pensée ? Cependant, pour mener à bien cet exercice, il n'était pas nécessaire de discréditer le point de vue philosophique en prétendant que toute interprétation du poème en termes de conceptualisation du verbe être ou d'étude de sa signification est anachronique car influencée par les conceptions de l'être développées plus tard par Platon et Aristote (p. 11-12). S'il est clair que la langue poétique de Parménide est caractéristique de l'époque archaïque, pourquoi faudrait-il en déduire que toute distinction de significations, d'emplois et de fonctions du verbe être serait chez lui inadéquate (p. 42) ? Ce présupposé, selon lequel tant le philosophe que le poète est entièrement tributaire de l'expression de son époque et incapable d'innover par rapport à elle, reste ininterrogé et injustifié, ainsi que la question de savoir quelles sont alors les conditions d'évolution d'une langue et d'une pensée.

La thèse de l'ouvrage est donc que Parménide est avant tout un poète qui joue avec la langue, qui exploite certains procédés linguistiques pour mettre en évidence l'importance syntaxique et morphologique du verbe «être ». Toute la première partie du poème, jusqu'au fragment 8 inclus, a une fonction introductive et exhortative, destinée à montrer que la polysémie de ce verbe et ses « variations paradigmatiques sans limites » sont indispensables pour comprendre le véritable contenu du poème, c'est-à-dire la partie cosmologique, et pour « assurer une assise infaillible à la parole même du poète-savant $\gg($ p. $41 ; i d$. p. 59). Ainsi, la revendication d'une vérité persuasive du discours ne repose pas sur la validité des démonstrations, 
soutenue par la rigueur des conjoncteurs logiques (il est d'ailleurs fréquent que les


l'être mais sur la nature en devenir, et si ce discours est qualifié par Parménide de trompeur, c'est que la tromperie est « la caractéristique essentielle de tout discours de persuasion $\gg$ (note 2 p. 145), à moins qu'il ne s'agisse que d'une apparence « qu'il ne tient qu'au destinataire de savoir déjouer » (p. 111-112).

Les procédés linguistiques révélant la richesse grammaticale du mot «être » sont, par exemple, l'alternance entre les verbes عĩvaı et $\pi \dot{\varepsilon} \lambda \varepsilon \iota \nu$, qui permet d'élargir le champ sémantique du verbe en ajoutant à la signification de «être là » celle de « circuler », de sorte que être «se définit comme une présence, ici et maintenant, mais en circulation permanente sur soi-même ; [...] comme un devenir immobile » (p. 64). Par ailleurs, la variante é $\mu \mu \varepsilon v \alpha \iota$ est traduite par « est résolument là », parce que cet infinitif contient en lui la racine de $\mu \dot{\varepsilon} v \varepsilon \iota \nu$, « demeurer » - et « peu importe » qu'il ne s'agisse pas d'un véritable rapport entre racines mais seulement d'une « apparence morphologique » (p. 66 et note 3 p. 161). Ensuite, $\pi \dot{\varepsilon} \lambda \varepsilon เ \nu$ est rapproché du है/ $\mu \pi \lambda \varepsilon \circ \nu$ du vers 8,24 , de manière à faire comprendre, par « décon-

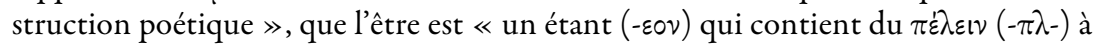
l'intérieur de lui $(\xi \mu) \gg($ p. 65). Enfin, le verbe $\pi \dot{\varepsilon} \lambda \varepsilon \imath \nu$ est encore rapproché de la racine $-\tau \varepsilon \lambda$ - des adjectifs signifiant la limite, de sorte que ces adjectifs à leur tour contiennent «la présence de l'être » et signalent « de façon cryptée que le verbe être est là » (p.68-69). Parce qu'il rayonne ainsi dans le mouvement de tout le poème, le verbe être est dit «le support d'un discours infailliblement vrai» (p.69).

Quant aux adjectifs exprimant la limite, ils constituent eux aussi un mouvement qui parcourt le fragment 8 , par les effets de résonance entre les vers 4 ( $\dot{\eta} \delta$ '


8, 4 est généralement corrigée par les éditeurs, parce qu'elle contredit les deux autres expressions. L'auteure propose au contraire de la conserver, en exploitant le jeu entre les deux valeurs de l'adjectif verbal, active et passive, et la proximité avec le sens actif du suffixe $-\theta$ po- de l'adjectif $\alpha \nu \dot{\lambda} \lambda \varepsilon \theta$ pov qui se trouve au vers précédent, tout cela devant mener à comprendre que l'alpha privatif sert à nier que $\dot{\sigma} \tau \iota$ aille « en s'achevant» (p. 24). Si donc l'auteure traduit l'expression par « et aussi inachevé », conservant l'apparente contradiction avec les deux autres expressions, elle invoque la «vigilance »du destinataire du poème (p. 31), qui comprendra que l'intention du poète était d'entendre par là «n'allant pas en s'achevant ». En effet, par contraste avec cette valeur active attribuée à $\dot{\alpha} \tau \dot{\varepsilon} \lambda \varepsilon \sigma \tau \circ \nu, \tau \varepsilon \tau \varepsilon \lambda \varepsilon \sigma \mu \dot{\varepsilon} \nu \circ \nu$ prendrait toute la charge passive, de sorte qu'ensemble ils signifieraient que $\dot{\varepsilon} \sigma \tau \iota$ ne peut s'achever parce qu'il est toujours déjà achevé. Quant au oủk ả $\tau \varepsilon \lambda \varepsilon \dot{\tau} \tau \eta \tau o v$ du vers 32, il serait «le centre vers lequel les deux expressions convergent », du fait qu'il possède lui-même un sens « aussi bien négatif que positif » (p. 29-31). En effet, les deux négations pourraient se renforcer plutôt que s'annuler car il est fréquent chez Homère que deux négations syntaxiques se renforcent. On remarquera cependant qu'il est vain d'invoquer l'autorité de Chantraine à l'appui d'une telle interprétation (note 2 p. 30) : à partir du fait, bien connu, que deux négations se renforcent lorsque la première est simple et la seconde composée, rien ne permet de déduire qu'il en va de même pour la négation d'un adjectif privatif; chez Homère comme 
partout ailleurs, dans ce cas les négations s'annulent. Dans sa traduction du poème, l'auteure conserve d'ailleurs le seul sens attesté de « pas inachevé », et l'on ne voit pas comment elle pourrait assumer un renforcement des deux négations, par exemple « tout à fait inachevé », sans devoir admettre un contresens. Certes, nous avons là une proposition nouvelle de résolution d'un problème textuel, mais il vaut la peine de se demander si la supposition de tels jeux de mots est vraiment une intervention plus légère que celle de corriger une lettre d'un manuscrit.

L'ouvrage apporte également une proposition nouvelle à la difficulté d'interpréter le $\dot{\varepsilon} \sigma \tau \iota$ affirmé par Parménide sans mention d'aucun sujet. L'auteure considère que cette forme verbale est le sujet même du fragment 8 , en tant que « représentant de toutes les autres formes », et exprimant «le sens plein d'être-là »

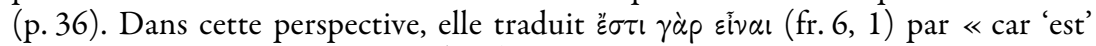

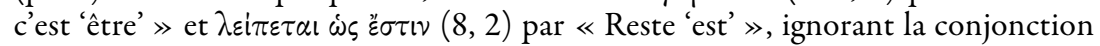
$\dot{\omega} \varsigma$, qui serait « une simple béquille syntaxique » (note 1 p. 74). Sans cet escamotage de la conjonction, il lui serait impossible de voir dans les vers 8, 1-2 une écriture « en boustrophédon », qui fait de है $\sigma \tau \iota \nu$ le sujet de $\lambda \varepsilon i \pi \varepsilon \tau \alpha$, alors que la lecture continue des deux vers révèle avec évidence que le sujet est le $\mu$ óvos $\mu \tilde{v} \theta 0 \varsigma$ ódoĩo du premier vers. Le seul argument pour cette nouvelle lecture est que, dans la suite du vers 2 , la préposition $\varepsilon \pi i$ i est placée derrière son régime, ce qu'on devrait plutôt juger bien ordinaire en langue archaïque (p. 74-75). Sans écarter la conjonction, il serait impossible d'affirmer que ce n'est pas un chemin qui reste, mais le $\dot{\varepsilon} \sigma \tau$ । lui-même, pour conclure que celui-ci est «le substrat à partir duquel la signification peut cheminer ». Quant à la traduction du vers $2,3: \dot{\eta} \mu \dot{\varepsilon} \nu$ ö $\pi \omega \varsigma$ है $\sigma \tau \iota \nu \tau \varepsilon$

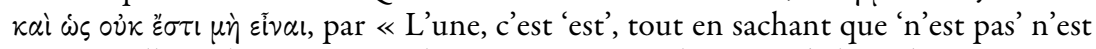
point », elle est laissée sans explication, ni quant à la raison de l'emphase parménidienne sur la dichotomie entre être et ne pas être, ni quant à la justification de la syntaxe, car comment justifier que $\dot{\omega} \varsigma$ soit suivi d'un infinitif, alors que ö $\pi \omega \varsigma$ est suivi de l'indicatif, et de même pour la structure parallèle du vers 5 ? Tout semble préférable à admettre que le verbe être puisse être utilisé en tant qu'expression impersonnelle ou modale, ce qui contredirait la thèse selon laquelle il a toujours une signification générale et indistincte. Ces procédés visent également à écarter les interprétations selon lesquelles il y a un sujet du $\dot{\sigma} \sigma \tau \iota$, d'abord implicite dans la forme verbale, et progressivement exprimé par le participe substantivé $\tau$ co żóv. Dans ces interprétations, cependant, le participe, loin de constituer une « quintessence ontologique » comme le prétend ironiquement l'auteure (p. 37), est un sujet tout aussi indéfini que celui de la forme conjuguée, sa signification étant limitée au seul fait d'être quelque chose qui est, de sorte que c'est toujours sur le fait d'être, et ensuite sur les « signes » qui l'accompagnent, que porte le message de Parménide.

Selon le présent ouvrage, au contraire, on l'a bien compris, le message consiste, d'abord et principalement, à assurer l'autorité de la parole du poète, et ensuite à « parler du monde réel dans son ensemble », dans la mesure où il « s'organise comme le kosmos linguistique que représente le poème »(p.112). Or, l'infaillibilité de la parole repose sur la constitution, par les interactions entre le je et le $t u$, d'une communauté de savoir qui met le destinataire dans la disposition d'en accepter la vérité. Ultimement, c'est le verbe être qui en constituerait la garantie, car il est le liant de cette communauté, « le lieu où les instances je et tu peuvent 
s'échanger indéfiniment » (p. 133). À défaut d'une explication précise de ce rôle de liant, une analogie est tracée avec l'élégie de Tyrtée, dans laquelle l'invocation de « tout homme quel qu'il soit » permet d'obtenir par avance l'accord de tout membre de la communauté des hommes. Mais comment l'invocation du verbe être pourrait-elle produire le même effet d'inclusion dans une communauté ? Nous devons à cet égard nous contenter de l'affirmation que le verbe être est la condition d'un « rapport simultané d'identité et de différence » entre le je et le $t u$, de sorte qu' « à leur origine semble encore, toujours et déjà se trouver l'essence linguistique du verbe être » (p. 135). On ne peut s'empêcher tout de même de se demander si ce montage linguistique n'est pas sensiblement plus anachronique que l'attribution à Parménide d'une réflexion sur la signification de « être ».

Pour jouer à notre tour avec les bouclages de boucles, nous conclurons que le lecteur du présent ouvrage qui s'estimerait insatisfait du manque de persuasion de l'argumentation n'aurait décidément rien compris à Parménide.

Annick STEVENS

Roberta IOLI (ed.), Gorgia. Testimonianze e frammenti. Introduzione, traduzione e commento, Rome, Carocci editore, 2013, 327 pages, ISBN 978-88-430-6892-0

Da alcuni anni si registra una crescita di interesse per Gorgia di Leontini e le sue opere, testimoniata dal buon numero di studi e pubblicazioni che con una certa continuità si offrono sia nell'ambito della filosofia che della cultura classica in generale; questo è senz'altro di buon auspicio per un avanzamento della comprensione del pensiero di questo intellettuale siceliota.

Il panorama italiano non è estraneo a questa tendenza, anzi si può dire che esso presenti un quadro ricchissimo di ricerche e studi di spessore: già solo guardando alle ultime due decadi si sono affermati numerosi studi nella letteratura critica anche internazionale ${ }^{1}$. Ora, a queste opere si aggiunge il volume a cura di Roberta Ioli per l'Editore Carocci nella collana Classici. Ioli, che aveva già presentato una traduzione del Peri tou me ontos di Gorgia nel 2010, in questo nuovo lavoro propone tutti i frammenti e le testimonianze gorgiane apportando alcune significative varianti rispetto all'edizione Diels-Kranz di suo riferimento (come esplicitato in chiusura nella «Nota al testo», p. 47).

Il lavoro è aperto da un ampio saggio introduttivo, «Gorgia e i "vortici della lingua” $\gg$, che intende mostrare la prospettiva ermeneutica della studiosa e che offre un'ampia analisi delle questioni che ruotano intorno al sofista. Ioli prende posizione nella disputa sulla figura di Gorgia che si è riproposta in maniera pressoché costante nel dibattito storiografico, incentrata sulla questione se Gorgia sia un filosofo o esclusivamente un retore, e sostiene che Gorgia è un filosofo-retore, in

1. Vd. L. Càffaro, Gorgia. Encomio di Elena, Apologia di Palamede, Firenze, Aletheia, 1997; G. Mazzara, Gorgia, la retorica del verosimile, Sankt Augustin, Academia Verlag, 1999; G. Paduano, Gorgia. Encomio di Elena, Napoli, Liguori, 2004; S. Mariani, In difesa di Palamede, Genova, Il Melangolo, 2010; S. Giombini, Gorgia epidittico. Commento filosofico all'Encomio di Elena, all'Apologia di Palamede, all' Epitaffio, Passignano s.T., Aguaplano, 2012; per le edizioni delle testimonianze e dei frammenti de I Sofisti cf. l'edizione di M. Bonazzi nella collana Bur, Milano 2007, e la riedizione di M. Untersteiner e A. M. Battegazzore, Milano, Bompiani, 2009. 